The $3^{\text {rd }}$ Conf. of SSFOP "Recent Techniques in Ornamental Plants Scope", Cairo, Egypt, 26/2/2017

Scientific J. Flowers \& Ornamental Plants

www.ssfop.com/journal

ISSN: 2356-7864

\title{
EFFECT OF PLANT DENSITY AND SOME VITAMINS, AS WELL AS, ACTIVE YEAST ON SWEET BASIL (OCIMUM BASILICUM) PLANT B- ESSENTIAL OIL PRODUCTION AND CHEMICAL CONSTITUENTS
}

\author{
M.A.H. Abdou, A.A. El-Sayed, R.A. Taha and Z.S.A. Marzok \\ Hort. Dept., Fac. Agric., Minia Univ., Egypt.
}

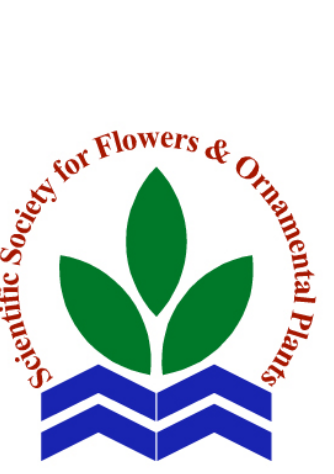

ABSTRACT: The main object of this study was to determine the effect of plant density and some vitamins and active yeast on essential oil production and some chemical constituents of sweet basil. The experiment was conducted during two successive seasons of 2014 and 2015 at the ornamental laboratory and nursery, Fac. Agric., Minia Univ. Results showed that by increasing plant densities. The oil production decreased/plant and increased/fed. Also, the lower density increased pigments and NPK \% elements.

All used vitamins and active yeast treatments increased essential oil production (oil \% and yield). The collection of vitamins increased Scientific J. Flowers \& essential oil \% and pigments content, while active yeast treatment Ornamental Plants, 4(1):101-120 (2017). increased essential oil yield and the percentages of $\mathrm{N}, \mathrm{P}$ and $\mathrm{K} \%$.

The best interaction treatments for essential oil production/plant

Received: 10/1/2017

Accepted:

31/1/2017 were two higher plant distance (80 and 65 plants $/ 14.5 \mathrm{~m}^{2}$ ) in combination with active yeast or 65 plants $/ 14.5 \mathrm{~m}^{2} \times$ vit. $\mathrm{B}_{1}+$ vit. $\mathrm{C}+$ vit. E. While, the highest yield of essential oil/fed were obtained with higher plant density (145 plants/14.5 $\left.\mathrm{m}^{2}\right)$ in combination with active yeast or vit. $B_{1}+$ vit. $C+$ vit. $E$.

Key words: Ocimum basilicum, plant density, vitamins, active yeast, essential oil, chemical constituents.

\section{INTRODUCTION}

Among various medicinal and culinary herbs, sweet basil is interested. Sweet basil (Ocimum basilicum, L.) from Lamiaceae family is one of the most common herbs. Essential oil of basil is known to posses antimicrobial, insecticidal activities and recently it has found to have vivo anti malaria activity (Bowes and Zheljazkov, 2004).

Khafi (2003) and Dadvand et al. (2009) found that density had significant effect on dry matter and essential oil yield of basil. Moreover, Arabasi and Bayran (2004) reported that the highest essential oil ratio obtained under non-nitrogen fertilizer condition with $20 \times 20 \mathrm{~cm}$ plant spacing.

\section{MATERIALS AND METHODS}

The experiment was carried out during the two seasons of 2014 and 2015 at the floriculture nursery and in the laboratory of floriculture, Fac. Agric., Minia Univ. to investigate the effect of plant density and active yeast, as well as, some vitamins and their interaction on essential oil and chemical composition of Ocimim basilicum, L. plants.

\section{Layout of the experiment:}

The experiment was arranged in a complete randomized block design in a split plot design with three replicates. The main 
plots (A) include four plant densities, which six treatments of active yeast and vitamins, as well as, control occupied the sub plots (B). Therefore, the interaction treatments $(A \times B)$ were 24 treatments. The experimental unit (plot) was $7.25 \times 2.0 \mathrm{~m}$ and containing 5 rows. The seedlings (at the stages of 4.5 leaves and 11-12 cm height) were cultivated in hills, therefore each plot contained 145, 105, 80 and 65 plants/14.5 $\mathrm{m}^{2}$ and distance between rows $40 \mathrm{~cm}$. The physical and chemical analysis of the used soil in both seasons are determined according to Jackson (1973) and shown in Table (a).

\section{Treatments:}

\section{Main plots (A)}

The main plots (A) included the following four plant densities:

1. 145 plants $/ 14.5 \mathrm{~m}^{2}=40000$ plants/fed (25 cm distance) and 29 plants/row.

2. 105 plants $/ 14.5 \mathrm{~m}^{2}=28966$ plants/fed (35 cm distance) and 21 plants/row.

3. 80 plants $/ 14.5 \mathrm{~m}^{2}=22069$ plants/fed (45 cm distance) and 16 plants/row.

4. 65 plants $/ 14.5 \mathrm{~m}^{2}=17931$ plants/fed (55 cm distance) and 13 plants/row.

\section{Sub plots (B)}

The sub plots (B) were devoted to six treatments as follows:
1. Control (spray with tap water).

2. Thiamine, vit. $B_{1}$ at $50 \mathrm{ppm}$.

3. Ascorbic acid, vit. C at $50 \mathrm{ppm}$.

4. Alpha tocopherol, vit. $\mathrm{E}$ at $10 \mathrm{ppm}$.

5. Vit. $\mathrm{B}_{1}+$ vit. $\mathrm{C}+$ vit. $\mathrm{E}$ at 50,50 and 10 ppm, respectively.

6. Active dry yeast (ADY) at $5 \mathrm{~g} / \mathrm{l}$.

Each of suspension of yeast and vitamins were applied by hand sprayer, 3 times. The first one was after 6 weeks from transplanting date (April $14^{\text {th }}$ ), the second one was added after two weeks from the first cut (May 28 $8^{\text {th }}$ ) and the third one was added after two week from the second cut (middle of July). The plants were sprayed till run off. All agricultural practices were performed as usual, in the region for the production of sweet basil plants.

\section{Harvesting times:}

During each experimental season, the plants were harvested three times at approximately $50 \%$ flowering. In each harvest, the plants were cut leaving about 10 $\mathrm{cm}$ above the soil surface. The first cut was done on $14^{\text {th }}$ of May, the second cut was done on $1^{\text {st }}$ of July. Meanwhile, the third cut was done on $28^{\text {th }}$ of August in the two growing seasons.

Table a. Physical and chemical properties of the used soil.

\begin{tabular}{|c|c|c|c|c|}
\hline Soil Character & Value & \multicolumn{2}{|c|}{ Soil Character } & Value \\
\hline Sand \% & 29.00 & \multicolumn{2}{|c|}{ Available P \% } & 14.98 \\
\hline Silt \% & 30.00 & \multicolumn{2}{|c|}{ Exch. $\mathrm{K}^{+}$(mg/100 g soil) } & 2.16 \\
\hline Clay \% & 41.00 & \multicolumn{2}{|c|}{ Exch. $\mathrm{Ca}^{++}(\mathrm{mg} / 100 \mathrm{~g}$ soil $)$} & 31.55 \\
\hline Texture grade & Clay loam & \multicolumn{2}{|c|}{ Exch. $\mathrm{Na}^{+}$(mg/100 g soil) } & 2.39 \\
\hline Organic matter \% & 1.68 & \multirow{5}{*}{$\begin{array}{l}\text { DTPA } \\
\text { Ext. ppm }\end{array}$} & $\mathbf{F e}$ & 7.55 \\
\hline $\mathrm{CaCO}_{3} \%$ & 2.08 & & $\mathbf{C u}$ & 2.16 \\
\hline E.C. (mmhos/cm) & 7.85 & & Zn & 2.64 \\
\hline pH (1:2.5) & 1.04 & & Mn & 7.35 \\
\hline Total N \% & 0.09 & & & \\
\hline
\end{tabular}




\section{Data recorded:}

The following data were recorded:

\section{Oil production}

Essential oil \% determination in random samples obtained from the air-dried herb of each treatment was carried out in each cut during the two experimental seasons according to the method described by British Pharmacopoeia (1963) by distilling $60 \mathrm{~g}$ of herb for 3 hours, in order to extract the essential oil and calculated essential oil yield/plant/cut and essential oil yield/plant and /fed.

\section{Chemical constituents}

The chlorophylls a, b and carotenoids were extracted by N-N dimethyl-formamnide according to Moran (1982), using the spectrophotometer at wave length of 656, 665 and $452.5 \mu \mathrm{m}$, respectively.

Nitrogen \% was determined by using the modified micro-kjeldahl method as described by Wilde et al. (1985).

Phosphorus \% was determined by the spectrophotometer at wave length of $650 \mu \mathrm{m}$ according to the method of Chapman and Pratt (1975).

Potassium \% was estimated using Flame-Photometry method according to Cottenie et al. (1982).

\section{Statistical analysis:}

The obtained data were tabulated and statistically analyzed according to MSTATC (1986) and the L.S.D. test at $5 \%$ was followed to compare between the means.

\section{RESULTS AND DISCUSSION}

\section{A- Essential oil production:}

\section{1- Essential oil \%:}

Data presented in Tables (1 and 2) indicated that the influence of density on essential oil \% become significant (at level of $5 \%$ ) increasing density decrease essential oil \% significantly during the three cuts in both seasons. Therefore, the lowest density
(65 plants/14.5 $\mathrm{m}^{2}$ ) results the highest percentage. It was observed that by increasing density, the essential oil \% decreased, which is in agreement with ElGendy et al. (2001) and Atghaei et al. (2015) on Ociminm basilicum.

All used treatments of vitamins (vit. $B_{1}$, vit. $\mathrm{C}$, vit. $\mathrm{E}$ and vit. $\mathrm{B}_{1}+$ vit. $\mathrm{C}+$ vit. E) and active yeast significantly increased essential oil \% during three cuts on both seasons comparing with control, except, vit. $\mathrm{B}_{1}$ in the second and third cuts in the second season. Among such five used treatments

The treatment of vit. $\mathrm{B}_{1}+$ vit. $\mathrm{C}+$ vit. $\mathrm{E}$ resulted the significant highest essential oil $\%$ comparing with other treatments and control. Similar results were obtained by Abd El-Salam (2014) on basil plants and Helmy (2016) on cumin plants.

The interaction between main and sub plots $(\mathrm{A} \times \mathrm{B})$ was significant for essential oil $\%$ during the three cuts in both seasons (Tables, 1 and 2). The highest values were obtained with the treatment of lowest density (65 plants $/ 14.5 \mathrm{~m}^{2}$ ) in combination with vit. $\mathrm{B}_{1}+$ vit. $\mathrm{C}+$ vit. $\mathrm{E}$ or vit. $\mathrm{E}$ or the interaction treatment of 80 plants $/ 14.5 \mathrm{~m}^{2} \times$ vit. $\mathrm{B}_{1}+$ vit. $\mathrm{C}+$ vit. E.

\section{2- Essential oil yield/plant/cut:}

Plant density had significant effect on essential oil yield/plant/cut in both seasons as clearly shown in Tables (3 and 4). Essential oil yield/plant/cut were increased by decreasing plant density. So, the treatment of 65 plants/14.5 $\mathrm{m}^{2}$ gave the highest essential oil yield/plant/cut in both seasons. Similar results were obtained by Khafi (2003) and Dadvand et al. (2009) on basil plants.

Data presented in Tables (2 and 3) indicated that all used five treatments of vitamins and active yeast significantly increased essential oil yield/plant/cut comparing with control in both seasons .

The treatment of active yeast had significantly the highest yield of essential oil 
M.A.H. Abdou et al.

Table 1. Effect of plant densities and some vitamins (vit. $B_{1}$, vit. $C$ and vit. $E$ ) and yeast treatments on essential oil percentage of sweet basil (Ocimum basilicum, L.) plants during the first season.

\begin{tabular}{|c|c|c|c|c|c|}
\hline \multicolumn{6}{|c|}{$1^{\text {st }}$ Cut } \\
\hline \multirow{2}{*}{ Treatments (B) } & \multicolumn{5}{|c|}{ Plant densities $/ 14.5 \mathrm{~m}^{2}$ (A) } \\
\hline & 145 & 105 & 80 & 65 & Mean (B) \\
\hline Control & 0.671 & 0.680 & 0.691 & 0.698 & 0.685 \\
\hline Vit. $B_{1}$ & 0.677 & 0.686 & 0.697 & 0.704 & 0.691 \\
\hline Vit. C & 0.688 & 0.697 & 0.708 & 0.715 & 0.702 \\
\hline Vit. E & 0.693 & 0.702 & 0.713 & 0.720 & 0.707 \\
\hline Vit. $B_{1}+$ Vit. C +Vit. E & 0.701 & 0.710 & 0.721 & 0.727 & 0.715 \\
\hline Yeast & 0.685 & 0.694 & 0.705 & 0.712 & 0.699 \\
\hline Mean (A) & 0.686 & 0.695 & 0.706 & 0.713 & 0.700 \\
\hline L.S.D. at $5 \%$ & \multicolumn{2}{|c|}{ A : 0.003} & B : 0.004 & \multicolumn{2}{|r|}{$\mathrm{AB}: 0.008$} \\
\hline \multicolumn{6}{|c|}{$2^{\text {nd }}$ Cut } \\
\hline Control & 0.686 & 0.693 & 0.701 & 0.707 & 0.697 \\
\hline Vit. $B_{1}$ & 0.693 & 0.700 & 0.708 & 0.714 & 0.704 \\
\hline Vit. C & 0.708 & 0.716 & 0.724 & 0.730 & 0.720 \\
\hline Vit. E & 0.713 & 0.721 & 0.729 & 0.735 & 0.725 \\
\hline Vit. $B_{1}+$ Vit. C+Vit. E & 0.722 & 0.730 & 0.738 & 0.744 & 0.734 \\
\hline Yeast & 0.701 & 0.708 & 0.716 & 0.722 & 0.712 \\
\hline Mean (A) & 0.704 & 0.711 & 0.719 & 0.725 & 0.715 \\
\hline L.S.D. at 5 \% & \multicolumn{2}{|c|}{ A : 0.004} & B : 0.005 & \multicolumn{2}{|r|}{$\mathrm{AB}: 0.010$} \\
\hline \multicolumn{6}{|c|}{$3^{\text {rd }}$ Cut } \\
\hline Control & 0.679 & 0.689 & 0.699 & 0.705 & 0.693 \\
\hline Vit. $B_{1}$ & 0.687 & 0.697 & 0.707 & 0.713 & 0.701 \\
\hline Vit. C & 0.698 & 0.708 & 0.718 & 0.724 & 0.712 \\
\hline Vit. E & 0.703 & 0.713 & 0.723 & 0.729 & 0.717 \\
\hline Vit. $B_{1}+$ Vit. C+Vit. E & 0.710 & 0.722 & 0.730 & 0.736 & 0.725 \\
\hline Yeast & 0.694 & 0.704 & 0.714 & 0.720 & 0.708 \\
\hline Mean (A) & 0.695 & 0.706 & 0.715 & 0.721 & 0.710 \\
\hline L.S.D. at 5 \% & \multicolumn{2}{|c|}{ A : 0.003} & B : 0.004 & \multicolumn{2}{|r|}{$\mathrm{AB}: 0.008$} \\
\hline
\end{tabular}


Table 2. Effect of plant densities and some vitamins (vit. $B_{1}$, vit. $C$ and vit. $E$ ) and yeast treatments on essential oil percentage of sweet basil (Ocimum basilicum, L.) plants during the second season.

$$
1^{\text {st }} \text { Cut }
$$

\begin{tabular}{|c|c|c|c|c|c|}
\hline \multirow{2}{*}{ Treatments (B) } & \multicolumn{5}{|c|}{ Plant densities $/ \mathbf{1 4 . 5} \mathrm{m}^{2}$ (A) } \\
\hline & 145 & 105 & 80 & 65 & Mean (B) \\
\hline Control & 0.677 & 0.682 & 0.689 & 0.697 & 0.686 \\
\hline Vit. $B_{1}$ & 0.683 & 0.689 & 0.693 & 0.704 & 0.692 \\
\hline Vit. C & 0.693 & 0.702 & 0.703 & 0.719 & 0.704 \\
\hline Vit. E & 0.698 & 0.707 & 0.718 & 0.726 & 0.712 \\
\hline Vit. $B_{1}+$ Vit. $C+$ Vit. E & 0.705 & 0.714 & 0.725 & 0.734 & 0.720 \\
\hline Yeast & 0.688 & 0.698 & 0.698 & 0.716 & 0.700 \\
\hline Mean (A) & 0.691 & 0.699 & 0.704 & 0.716 & 0.703 \\
\hline L.S.D. at $5 \%$ & \multicolumn{2}{|c|}{ A : 0.003} & B : 0.005 & \multicolumn{2}{|r|}{$\mathrm{AB}: 0.010$} \\
\hline \multicolumn{6}{|c|}{$2^{\text {nd }}$ Cut } \\
\hline Control & 0.696 & 0.701 & 0.708 & 0.715 & 0.705 \\
\hline Vit. $\mathbf{B}_{1}$ & 0.697 & 0.705 & 0.714 & 0.720 & 0.709 \\
\hline Vit. C & 0.712 & 0.721 & 0.729 & 0.734 & 0.724 \\
\hline Vit. E & 0.718 & 0.725 & 0.734 & 0.741 & 0.730 \\
\hline Vit. $B_{1}+$ Vit. C+Vit. E & 0.777 & 0.733 & 0.742 & 0.750 & 0.738 \\
\hline Yeast & 0.704 & 0.713 & 0.720 & 0.726 & 0.716 \\
\hline Mean (A) & 0.709 & 0.716 & 0.725 & 0.731 & 0.720 \\
\hline L.S.D. at $5 \%$ & \multicolumn{2}{|c|}{ A : 0.004} & B : 0.006 & \multicolumn{2}{|r|}{$\mathrm{AB}: 0.012$} \\
\hline \multicolumn{6}{|c|}{$3^{\text {rd }}$ Cut } \\
\hline Control & 0.686 & 0.697 & 0.705 & 0.714 & 0.701 \\
\hline Vit. $B_{1}$ & 0.689 & 0.699 & 0.710 & 0.716 & 0.704 \\
\hline Vit. C & 0.701 & 0.701 & 0.721 & 0.726 & 0.712 \\
\hline Vit. E & 0.709 & 0.718 & 0.729 & 0.735 & 0.723 \\
\hline Vit. $B_{1}+$ Vit. C+Vit. E & 0.717 & 0.725 & 0.737 & 0.744 & 0.731 \\
\hline Yeast & 0.697 & 0.708 & 0.717 & 0.722 & 0.711 \\
\hline Mean (A) & 0.700 & 0.708 & 0.720 & 0.726 & 0.714 \\
\hline L.S.D. at $5 \%$ & \multicolumn{2}{|c|}{ A : 0.005} & B : 0.005 & \multicolumn{2}{|r|}{$\mathrm{AB}: 0.010$} \\
\hline
\end{tabular}


M.A.H. Abdou et al.

Table 3. Effect of plant densities and some vitamins (vit. $B_{1}$, vit. $C$ and vit. $E$ ) and yeast treatments on essential oil yield/plant/cut (ml) of sweet basil (Ocimum basilicum, $\mathrm{L}$.) plants during the first season.

\begin{tabular}{|c|c|c|c|c|c|}
\hline \multicolumn{6}{|c|}{$\mathbf{1}^{\text {st }}$ Cut } \\
\hline \multirow{2}{*}{ Treatments (B) } & \multicolumn{5}{|c|}{ Plant densities $/ 14.5 \mathrm{~m}^{2}$ (A) } \\
\hline & 145 & 105 & 80 & 65 & Mean (B) \\
\hline Control & 0.225 & 0.218 & 0.296 & 0.349 & 0.272 \\
\hline Vit. $B_{1}$ & 0.265 & 0.367 & 0.410 & 0.422 & 0.365 \\
\hline Vit. C & 0.303 & 0.421 & 0.440 & 0.508 & 0.417 \\
\hline Vit. E & 0.287 & 0.404 & 0.431 & 0.430 & 0.388 \\
\hline Vit. $B_{1}+$ Vit. C +Vit. E & 0.470 & 0.541 & 0.638 & 0.653 & 0.575 \\
\hline Yeast & 0.499 & 0.569 & 0.678 & 0.726 & 0.617 \\
\hline Mean (A) & 0.341 & 0.419 & 0.481 & 0.514 & 0.438 \\
\hline L.S.D. at $5 \%$ & \multicolumn{2}{|c|}{ A : 0.081} & B : 0.037 & \multicolumn{2}{|r|}{$\mathrm{AB}: 0.074$} \\
\hline \multicolumn{6}{|c|}{$2^{\text {nd }}$ Cut } \\
\hline Control & 0.250 & 0.244 & 0.314 & 0.517 & 0.330 \\
\hline Vit. $B_{1}$ & 0.321 & 0.432 & 0.518 & 0.674 & 0.485 \\
\hline Vit. C & 0.352 & 0.433 & 0.533 & 0.756 & 0.517 \\
\hline Vit. E & 0.344 & 0.452 & 0.507 & 0.807 & 0.526 \\
\hline Vit. $B_{1}+$ Vit. C+Vit. E & 0.491 & 0.824 & 0.856 & 0.954 & 0.780 \\
\hline Yeast & 0.541 & 0.787 & 0.927 & 0.945 & 0.799 \\
\hline Mean (A) & 0.382 & 0.526 & 0.607 & 0.773 & 0.571 \\
\hline L.S.D. at $5 \%$ & \multicolumn{2}{|c|}{ A : 0.039} & B : 0.040 & \multicolumn{2}{|r|}{$\mathrm{AB}: 0.080$} \\
\hline \multicolumn{6}{|c|}{$3^{\text {rd }}$ Cut } \\
\hline Control & 0.338 & 0.404 & 0.414 & 0.533 & 0.421 \\
\hline Vit. $B_{1}$ & 0.387 & 0.602 & 0.637 & 0.974 & 0.648 \\
\hline Vit. C & 0.510 & 0.648 & 0.693 & 1.073 & 0.729 \\
\hline Vit. E & 0.439 & 0.629 & 0.647 & 1.053 & 0.689 \\
\hline Vit. $B_{1}+$ Vit. C+Vit. E & 0.957 & 0.993 & 1.011 & 1.281 & 1.003 \\
\hline Yeast & 0.949 & 0.872 & 1.271 & 1.440 & 1.130 \\
\hline Mean (A) & 0.590 & 0.690 & 0.777 & 1.023 & 0.769 \\
\hline L.S.D. at $5 \%$ & \multicolumn{2}{|c|}{ A : 0.080} & B : 0.092 & \multicolumn{2}{|r|}{$\mathrm{AB}: 0.184$} \\
\hline
\end{tabular}


Table 4. Effect of plant densities and some vitamins (vit. $B_{1}$, vit. $C$ and vit. $E$ ) and yeast treatments on essential oil yield/plant/cut (ml) of sweet basil (Ocimum basilicum, L.) plants during the second season.

\begin{tabular}{|c|c|c|c|c|c|}
\hline \multicolumn{6}{|c|}{$\mathbf{1}^{\text {st }}$ Cut } \\
\hline \multirow{2}{*}{ Treatments (B) } & \multicolumn{5}{|c|}{ Plant densities $/ \mathbf{1 4 . 5} \mathrm{m}^{2}$ (A) } \\
\hline & 145 & 105 & 80 & 65 & Mean (B) \\
\hline Control & 0.259 & 0.129 & 0.335 & 0.384 & 0.276 \\
\hline Vit. $B_{1}$ & 0.284 & 0.381 & 0.396 & 0.416 & 0.369 \\
\hline Vit. C & 0.312 & 0.444 & 0.452 & 0.566 & 0.442 \\
\hline Vit. E & 0.301 & 0.395 & 0.421 & 0.438 & 0.388 \\
\hline Vit. $B_{1}+$ Vit. C +Vit. E & 0.367 & 0.596 & 0.633 & 0.667 & 0.565 \\
\hline Yeast & 0.430 & 0.616 & 0.669 & 0.740 & 0.612 \\
\hline Mean (A) & 0.159 & 0.481 & 0.540 & 0.591 & 0.441 \\
\hline L.S.D. at $5 \%$ & \multicolumn{2}{|c|}{ A : 0.096} & B : 0.037 & \multicolumn{2}{|r|}{$\mathrm{AB}: 0.074$} \\
\hline \multicolumn{6}{|c|}{$2^{\text {nd }}$ Cut } \\
\hline Control & 0.381 & 0.336 & 0.393 & 0.459 & 0.392 \\
\hline Vit. $B_{1}$ & 0.447 & 0.631 & 0.656 & 0.707 & 0.609 \\
\hline Vit. C & 0.489 & 0.679 & 0.724 & 0.761 & 0.662 \\
\hline Vit. E & 0.470 & 0.661 & 0.672 & 0.759 & 0.640 \\
\hline Vit. $B_{1}+$ Vit. C+Vit. E & 0.810 & 0.986 & 1.027 & 1.043 & 0.953 \\
\hline Yeast & 0.778 & 1.078 & 1.148 & 1.229 & 1.057 \\
\hline Mean (A) & 0.383 & 0.783 & 0.827 & 0.883 & 0.717 \\
\hline L.S.D. at $5 \%$ & \multicolumn{2}{|c|}{ A : 0.105} & B : 0.041 & \multicolumn{2}{|r|}{$\mathrm{AB}: 0.082$} \\
\hline \multicolumn{6}{|c|}{$3^{\text {rd }}$ Cut } \\
\hline Control & 0.423 & 0.435 & 0.505 & 0.585 & 0.487 \\
\hline Vit. $\mathbf{B}_{1}$ & 0.669 & 0.816 & 0.896 & 0.948 & 0.832 \\
\hline Vit. C & 0.735 & 0.852 & 0.957 & 1.047 & 0.896 \\
\hline Vit. E & 0.708 & 0.856 & 0.940 & 1.001 & 0.875 \\
\hline Vit. $B_{1}+$ Vit. C+Vit. E & 1.080 & 1.229 & 1.305 & 1.351 & 1.240 \\
\hline Yeast & 1.004 & 1.446 & 1.499 & 1.574 & 1.378 \\
\hline Mean (A) & 0.768 & 0.993 & 1.072 & 1.140 & 0.941 \\
\hline L.S.D. at $5 \%$ & \multicolumn{2}{|c|}{ A : 0.098} & B : 0.038 & \multicolumn{2}{|r|}{$\mathrm{AB}: 0.076$} \\
\hline
\end{tabular}


during the three cuts in both seasons, except the second cut during the first season. Salman (2006), Abdou et al. (2014) and Omar et al. (2016) found that active yeast treatment had positive effects on essential oil yield of basil plants.

The interaction between main and sub plots was significant for essential oil yield/plant/cut in both seasons. Generally, the highest yield of essential oil/plant/cut were obtained by the treatments of 80 or 65 plannts $/ 14.5 \mathrm{~m}^{2}$ in combination with active yeast or cultivated plants at 65 plants/14.5 $\mathrm{m}^{2}$ and sprayed with vit. $\mathrm{B}_{1}+$ vit. $\mathrm{C}+$ vit. $\mathrm{E}$ (Tables, 3 and 4).

\section{3- Essential oil yield/plant and /fed:}

Essential oil yield per plant was significant increase (1.313. 1.635, 1.865 and $2.311 \mathrm{ml} /$ plant) with decreasing plant density $\left(145,105,80\right.$ and 65 plants $\left./ 14.5 \mathrm{~m}^{2}\right)$ in the first season. The same trend was obtained in the second season. The opposite trend was obtained for essential oil yield/fed where by increasing the density, essential oil yield/fed increases. The highest yield of essential oil (52.86 and 66.29 liter/fed in both seasons, respectively) were obtained by the treatment of higher density (40000 plants/fed) as compared with other treatments (28966, 22069 and 17931 plants/fed) which were recorded 47.51, 41.53 and 41.28 liter/fed in the first season and 60.67, 50.23 and 48.36 liter/fed in the second season, respectively.

It could be said that by increasing the distance between plants the growth of a single plant increases because of decreasing the competition for absorbing light, water and nutrition. But increasing growth due to that, could not compensate for increase in growth tissue resulting from the number of plants in unit, as a result of higher density the yield of dry matter increases, that reflected in essential oil yield/fed Similar results were obtained by Ram et al. (2002) and Arabasi and Bayran (2004) on Ocimum spp. and Katar and Gurbuz (2008) on Melissa officinalis.
Concerning the effect of vitamins and active yeast treatments, data in Tables (5 and 6 ) showed that the best treatments which gave the highest essential oil yield/plant and /fed was active yeast followed by vit. $\mathrm{B}_{1}+$ vit. $\mathrm{C}+$ vit. $\mathrm{E}$ which significant differences between such two superior treatments. Active yeast had positive effect on essential oil yield because its contain many components which enhance and stimulate essential oil production. Similar results were obtained by Salman (2006), Abdou et al. (2014), Nassar et al. (2015) and Omar et al. (2016) on Ocimum sp.

The interaction between main and sub plots was significant for essential oil yield/plant and /fed in both seasons. The best interaction treatments for essential oil yield /plant were obtained by the two densities 65 and 80 plants $/ 14.5 \mathrm{~m}^{2}$ in combination with active yeast in both seasons and density in 65 plants $/ 14.5 \mathrm{~m}^{2}$ in combination with vit. $\mathrm{B}_{1}+$ vit. C + vit. E as clearly shown in Table (5). The highest yield of essential oil/fed were obtained by cultivated plants in higher density 145 plants $/ 14.5 \mathrm{~m}^{2}$ in combination with active yeast or vit. $\mathrm{B}_{1}+$ vit. $\mathrm{C}+$ vit. $\mathrm{E}$ in both seasons or plant density 105 plants/14.5 $\mathrm{m}^{2}$ in combination with active yeast during the second season as clearly shown in Table (6).

\section{B- Chemical constituents:}

\section{1- Photosynthetic pigments:}

Data presented in Tables (7 to 12) showed that the effect of plant densities on photosynthetic pigments (chl. a, b and carotenoids) was significant during the three cuts in both seasons. The significantly highest contents of chl. a, b and carotenoids were obtained with the lowest density treatment (65 plants/14.5 m²). Similar results were obtained by Badran and Hafez (2002) on Nigella sativa and Badran et al. (2003 and 2007) on Pimpinella anisum and fennel plants, respectively.

Regarding the effect of some vitamins and active yeast treatments, data in Tables (7 to 12 ) showed that all five used treatments 
Table 5. Effect of plant densities and some vitamins (vit. $B_{1}$, vit. $C$ and vit. $E$ ) and yeast treatments on essential oil yield/plant/season ( $\mathrm{ml} /$ plant) of sweet basil (Ocimum basilicum, L.) plants during two seasons.

\begin{tabular}{|c|c|c|c|c|c|}
\hline \multicolumn{6}{|c|}{ First season } \\
\hline \multirow{2}{*}{ Treatments (B) } & \multicolumn{5}{|c|}{ Plant densities $/ 14.5 \mathrm{~m}^{2}(\mathrm{~A})$} \\
\hline & 145 & 105 & 80 & 65 & Mean (B) \\
\hline Control & 0.813 & 0.867 & 1.024 & 1.399 & 1.023 \\
\hline Vit. $B_{1}$ & 0.974 & 1.401 & 1.565 & 2.071 & 1.498 \\
\hline Vit. C & 1.166 & 1.502 & 1.666 & 2.337 & 1.663 \\
\hline Vit. E & 1.070 & 1.486 & 1.585 & 2.290 & 1.603 \\
\hline Vit. $B_{1}+$ Vit. C+Vit. E & 1.917 & 2.357 & 2.506 & 2.687 & 2.358 \\
\hline Yeast & 1.989 & 2.228 & 2.876 & 3.112 & 2.546 \\
\hline Mean (A) & 1.313 & 1.635 & 1.865 & 2.311 & \\
\hline L.S.D. at $5 \%$ & \multicolumn{2}{|c|}{ A : 0.131} & B : 0.121 & \multicolumn{2}{|r|}{$\mathrm{AB}: 0.242$} \\
\hline \multicolumn{6}{|c|}{ Second season } \\
\hline Control & 1.062 & 0.901 & 1.234 & 1.429 & 1.155 \\
\hline Vit. $B_{1}$ & 1.400 & 1.828 & 1.948 & 2.072 & 1.810 \\
\hline Vit. C & 1.536 & 1.975 & 2.133 & 2.374 & 2.000 \\
\hline Vit. E & 1.479 & 1.912 & 2.033 & 2.198 & 1.903 \\
\hline Vit. $B_{1}+$ Vit. $C+$ Vit. $E$ & 2.256 & 2.811 & 2.965 & 3.061 & 2.758 \\
\hline Yeast & 2.211 & 3.141 & 3.316 & 3.543 & 3.047 \\
\hline Mean (A) & 1.310 & 2.258 & 2.439 & 2.615 & \\
\hline L.S.D. at $5 \%$ & \multicolumn{2}{|c|}{ A : 0.142} & B : 0.135 & & $\mathrm{AB}: 0.270$ \\
\hline
\end{tabular}

Table 6. Effect of plant densities and some vitamins (vit. $B_{1}$, vit. $C$ and vit. $E$ ) and yeast treatments on essential oil yield/fed/season (liter/fed) of sweet basil (Ocimum basilicum, L.) plants during two seasons.

\begin{tabular}{|c|c|c|c|c|c|}
\hline \multicolumn{6}{|c|}{ First season } \\
\hline \multirow{2}{*}{ Treatments (B) } & \multicolumn{5}{|c|}{ Plant densities $/ 14.5 \mathrm{~m}^{2}$ (A) } \\
\hline & 145 & 105 & 80 & 65 & Mean (B) \\
\hline Control & 32.52 & 25.11 & 22.60 & 25.09 & 26.33 \\
\hline Vit. $B_{1}$ & 38.96 & 40.58 & 34.54 & 37.14 & 37.81 \\
\hline Vit. C & 46.64 & 43.51 & 36.77 & 41.90 & 42.21 \\
\hline Vit. E & 42.80 & 43.04 & 34.98 & 41.06 & 40.47 \\
\hline Vit. $B_{1}+$ Vit. C+Vit. E & 76.68 & 68.27 & 55.30 & 48.18 & 62.11 \\
\hline Yeast & 79.56 & 64.54 & 63.47 & 55.80 & 65.84 \\
\hline Mean (A) & 52.86 & 47.51 & 41.53 & 41.28 & \\
\hline L.S.D. at $5 \%$ & & & B : 2.23 & & $\mathrm{AB}: 4.46$ \\
\hline \multicolumn{6}{|c|}{ Second season } \\
\hline Control & 42.48 & 26.10 & 27.23 & 25.62 & 30.36 \\
\hline Vit. $B_{1}$ & 56.00 & 52.95 & 42.99 & 37.15 & 47.27 \\
\hline Vit. C & 61.44 & 57.21 & 47.07 & 42.57 & 52.07 \\
\hline Vit. E & 59.16 & 55.38 & 44.87 & 39.41 & 49.71 \\
\hline Vit. $B_{1}+$ Vit. C+Vit. E & 90.24 & 81.42 & 65.43 & 54.89 & 72.99 \\
\hline Yeast & 88.44 & 90.98 & 73.18 & 63.53 & 79.03 \\
\hline Mean (A) & 66.29 & 60.67 & 50.23 & 48.36 & \\
\hline L.S.D. at $5 \%$ & \multicolumn{2}{|c|}{ A : 2.68} & B : 2.57 & & $\mathrm{AB}: 5.14$ \\
\hline
\end{tabular}


M.A.H. Abdou et al.

Table 7. Effect of plant densities and some vitamins (vit. $B_{1}$, vit. $C$ and vit. $E$ ) and yeast treatments on chlorophyll a of sweet basil (Ocimum basilicum, L.) plants during the first season.

\begin{tabular}{|c|c|c|c|c|c|}
\hline \multicolumn{6}{|c|}{$1^{\text {st }}$ Cut } \\
\hline \multirow{2}{*}{ Treatments (B) } & \multicolumn{5}{|c|}{ Plant densities $/ 14.5 \mathrm{~m}^{2}$ (A) } \\
\hline & 145 & 105 & 80 & 65 & Mean (B) \\
\hline Control & 2.220 & 2.255 & 2.280 & 2.299 & 2.264 \\
\hline Vit. $B_{1}$ & 2.250 & 2.280 & 2.303 & 2.329 & 2.291 \\
\hline Vit. C & 2.287 & 2.320 & 2.345 & 2.370 & 2.331 \\
\hline Vit. E & 2.300 & 2.336 & 2.360 & 2.390 & 2.347 \\
\hline Vit. $B_{1}+$ Vit. $C+$ Vit. E & 2.315 & 2.351 & 2.375 & 2.406 & 2.362 \\
\hline Yeast & 2.270 & 2.301 & 2.322 & 2.348 & 2.310 \\
\hline Mean (A) & 2.274 & 2.307 & 2.331 & 2.357 & 2.317 \\
\hline L.S.D. at $5 \%$ & \multicolumn{2}{|c|}{ A : 0.010} & B : 0.008 & \multicolumn{2}{|r|}{$\mathrm{AB}: 0.016$} \\
\hline \multicolumn{6}{|c|}{$2^{\text {nd }}$ Cut } \\
\hline Control & 0.270 & 0.290 & 2.315 & 2.340 & 2.304 \\
\hline Vit. $B_{1}$ & 2.285 & 2.325 & 2.348 & 2.371 & 2.332 \\
\hline Vit. C & 2.322 & 2.360 & 2.375 & 2.401 & 2.365 \\
\hline Vit. E & 2.339 & 2.375 & 2.390 & 2.409 & 2.378 \\
\hline Vit. $B_{1}+$ Vit. C+Vit. E & 2.355 & 2.399 & 2.410 & 2.430 & 2.399 \\
\hline Yeast & 2.301 & 2.346 & 2.360 & 2.385 & 2.348 \\
\hline Mean (A) & 2.312 & 2.349 & 2.366 & 2.389 & 2.354 \\
\hline L.S.D. at $5 \%$ & \multicolumn{2}{|c|}{ A : 0.015} & B : 0.011 & \multicolumn{2}{|r|}{$\mathrm{AB}: 0.022$} \\
\hline \multicolumn{6}{|c|}{$3^{\text {rd }}$ Cut } \\
\hline Control & 2.240 & 2.278 & 2.300 & 2.324 & 2.286 \\
\hline Vit. $B_{1}$ & 2.270 & 2.301 & 2.321 & 2.349 & 2.310 \\
\hline Vit. C & 2.301 & 2.336 & 2.350 & 2.380 & 2.342 \\
\hline Vit. E & 2.321 & 2.353 & 2.370 & 2.400 & 2.361 \\
\hline Vit. $B_{1}+$ Vit. C+Vit. E & 2.340 & 2.375 & 2.399 & 2.412 & 2.382 \\
\hline Yeast & 2.285 & 2.318 & 2.336 & 2.365 & 2.326 \\
\hline Mean (A) & 2.293 & 2.327 & 2.346 & 3.372 & 2.335 \\
\hline L.S.D. at $5 \%$ & \multicolumn{2}{|c|}{ A : 0.012} & B : 0.007 & \multicolumn{2}{|r|}{ AB : 0.014} \\
\hline
\end{tabular}


Table 8. Effect of plant densities and some vitamins (vit. $B_{1}$, vit. $C$ and vit. $E$ ) and yeast treatments on chlorophyll a of sweet basil (Ocimum basilicum, L.) plants during the second season.

\begin{tabular}{|c|c|c|c|c|c|}
\hline \multicolumn{6}{|c|}{$1^{\text {st }}$ Cut } \\
\hline \multirow{2}{*}{ Treatments (B) } & \multicolumn{5}{|c|}{ Plant densities $/ 14.5 \mathrm{~m}^{2}$ (A) } \\
\hline & 145 & 105 & 80 & 65 & Mean (B) \\
\hline Control & 2.287 & 2.323 & 2.348 & 2.368 & 2.332 \\
\hline Vit. $B_{1}$ & 2.318 & 2.348 & 2.372 & 2.399 & 2.360 \\
\hline Vit. C & 2.356 & 2.390 & 2.415 & 2.441 & 2.401 \\
\hline Vit. E & 2.369 & 2.406 & 2.431 & 2.462 & 2.417 \\
\hline Vit. $B_{1}+$ Vit. $C+$ Vit. E & 2.384 & 2.422 & 2.446 & 2.478 & 2.433 \\
\hline Yeast & 2.338 & 2.370 & 2.392 & 2.418 & 2.379 \\
\hline Mean (A) & 2.342 & 2.376 & 2.401 & 2.428 & 2.387 \\
\hline L.S.D. at $5 \%$ & \multicolumn{2}{|c|}{ A :0.013 } & B : 0.009 & \multicolumn{2}{|r|}{ AB : 0.018} \\
\hline \multicolumn{6}{|c|}{$2^{\text {nd }}$ Cut } \\
\hline Control & 0.278 & 0.299 & 2.384 & 2.410 & 2.373 \\
\hline Vit. $B_{1}$ & 2.354 & 2.395 & 2.418 & 2.442 & 2.402 \\
\hline Vit. C & 2.392 & 2.431 & 2.446 & 2.473 & 2.436 \\
\hline Vit. E & 2.409 & 2.446 & 2.462 & 2.481 & 2.449 \\
\hline Vit. $B_{1}+$ Vit. C+Vit. E & 2.426 & 2.471 & 2.482 & 2.503 & 2.471 \\
\hline Yeast & 2.370 & 2.416 & 2.431 & 2.457 & 2.418 \\
\hline Mean (A) & 2.381 & 2.419 & 2.437 & 2.461 & 2.425 \\
\hline L.S.D. at $5 \%$ & \multicolumn{2}{|c|}{ A : 0.017} & B : 0.012 & \multicolumn{2}{|r|}{ AB : 0.024} \\
\hline \multicolumn{6}{|c|}{$3^{\text {rd }}$ Cut } \\
\hline Control & 2.307 & 2.346 & 2.369 & 2.394 & 2.355 \\
\hline Vit. $B_{1}$ & 2.338 & 2.370 & 2.391 & 2.419 & 2.379 \\
\hline Vit. C & 2.370 & 2.406 & 2.421 & 2.451 & 2.412 \\
\hline Vit. E & 2.391 & 2.424 & 2.441 & 2.472 & 2.432 \\
\hline Vit. $B_{1}+$ Vit. C+Vit. E & 2.410 & 2.446 & 2.471 & 2.484 & 2.453 \\
\hline Yeast & 2.354 & 2.388 & 2.406 & 2.436 & 2.396 \\
\hline Mean (A) & 2.362 & 2.397 & 2.416 & 3.473 & 2.405 \\
\hline L.S.D. at $5 \%$ & \multicolumn{2}{|c|}{ A : 0.014} & B : 0.007 & \multicolumn{2}{|r|}{$\mathrm{AB}: 0.014$} \\
\hline
\end{tabular}


M.A.H. Abdou et al.

Table 9. Effect of plant densities and some vitamins (vit. $B_{1}$, vit. $C$ and vit. $E$ ) and yeast treatments on chlorophyll b of sweet basil (Ocimum basilicum, L.) plants during the first season.

\begin{tabular}{|c|c|c|c|c|c|}
\hline \multicolumn{6}{|c|}{$1^{\text {st }}$ Cut } \\
\hline \multirow{2}{*}{ Treatments (B) } & \multicolumn{5}{|c|}{ Plant densities $/ 14.5 \mathrm{~m}^{2}$ (A) } \\
\hline & 145 & 105 & 80 & 65 & Mean (B) \\
\hline Control & 0.730 & 0.742 & 0.750 & 0.756 & 0.745 \\
\hline Vit. $B_{1}$ & 0.741 & 0.750 & 0.756 & 0.766 & 0.753 \\
\hline Vit. C & 0.752 & 0.763 & 0.771 & 0.780 & 0.797 \\
\hline Vit. E & 0.756 & 0.768 & 0.786 & 0.796 & 0.772 \\
\hline Vit. $B_{1}+$ Vit. $C+$ Vit. E & 0.761 & 0.773 & 0.792 & 0.797 & 0.781 \\
\hline Yeast & 0.746 & 0.757 & 0.762 & 0.773 & 0.760 \\
\hline Mean (A) & 0.748 & 0.759 & 0.769 & 0.776 & 0.763 \\
\hline L.S.D. at $5 \%$ & \multicolumn{2}{|c|}{ A : 0.005} & B : 0.004 & \multicolumn{2}{|r|}{$\mathrm{AB}: 0.008$} \\
\hline \multicolumn{6}{|c|}{$2^{\text {nd }}$ Cut } \\
\hline Control & 0.746 & 0.753 & 0.761 & 0.770 & 0.758 \\
\hline Vit. $B_{1}$ & 0.751 & 0.765 & 0.772 & 0.780 & 0.767 \\
\hline Vit. C & 0.764 & 0.776 & 0.782 & 0.790 & 0.778 \\
\hline Vit. E & 0.769 & 0.781 & 0.786 & 0.793 & 0.782 \\
\hline Vit. $B_{1}+$ Vit. C+Vit. E & 0.775 & 0.789 & 0.793 & 0.800 & 0.789 \\
\hline Yeast & 0.757 & 0.772 & 0.776 & 0.785 & 0.773 \\
\hline Mean (A) & 0.760 & 0.773 & 0.778 & 0.786 & 0.774 \\
\hline L.S.D. at $5 \%$ & \multicolumn{2}{|c|}{ A : 0.003} & B : 0.004 & \multicolumn{2}{|r|}{$\mathrm{AB}: 0.008$} \\
\hline \multicolumn{6}{|c|}{$3^{\text {rd }}$ Cut } \\
\hline Control & 0.736 & 0.749 & 0.757 & 0.764 & 0.752 \\
\hline Vit. $B_{1}$ & 0.746 & 0.757 & 0.762 & 0.773 & 0.760 \\
\hline Vit. C & 0.757 & 0.768 & 0.773 & 0.783 & 0.770 \\
\hline Vit. E & 0.763 & 0.774 & 0.780 & 0.791 & 0.777 \\
\hline Vit. $B_{1}+$ Vit. C+Vit. E & 0.770 & 0.781 & 0.789 & 0.794 & 0.784 \\
\hline Yeast & 0.751 & 0.762 & 0.768 & 0.778 & 0.765 \\
\hline Mean (A) & 0.754 & 0.765 & 0.772 & 0.781 & 0.768 \\
\hline L.S.D. at $5 \%$ & \multicolumn{2}{|c|}{ A : 0.003} & B : 0.003 & \multicolumn{2}{|r|}{$\mathrm{AB}: 0.006$} \\
\hline
\end{tabular}


Table 10. Effect of plant densities and some vitamins (vit. $B_{1}$, vit. $C$ and vit. E) and yeast treatments on chlorophyll b of sweet basil (Ocimum basilicum, L.) plants during the second season.

\begin{tabular}{|c|c|c|c|c|c|}
\hline \multicolumn{6}{|c|}{$\mathbf{1}^{\text {st }}$ Cut } \\
\hline \multirow{2}{*}{ Treatments (B) } & \multicolumn{5}{|c|}{ Plant densities $/ 14.5 \mathrm{~m}^{2}$ (A) } \\
\hline & 145 & 105 & 80 & 65 & Mean (B) \\
\hline Control & 0.752 & 0.764 & 0.773 & 0.779 & 0.767 \\
\hline Vit. $B_{1}$ & 0.763 & 0.773 & 0.779 & 0.789 & 0.776 \\
\hline Vit. C & 0.775 & 0.786 & 0.794 & 0.803 & 0.821 \\
\hline Vit. E & 0.779 & 0.791 & 0.810 & 0.820 & 0.795 \\
\hline Vit. $B_{1}+$ Vit. C +Vit. E & 0.784 & 0.796 & 0.816 & 0.821 & 0.804 \\
\hline Yeast & 0.768 & 0.780 & 0.785 & 0.796 & 0.783 \\
\hline Mean (A) & 0.770 & 0.782 & 0.792 & 0.799 & 0.786 \\
\hline L.S.D. at $5 \%$ & \multicolumn{2}{|c|}{ A : 0.006} & B : 0.005 & \multicolumn{2}{|r|}{$\mathrm{AB}: 0.010$} \\
\hline \multicolumn{6}{|c|}{$2^{\text {nd }}$ Cut } \\
\hline Control & 0.768 & 0.776 & 0.784 & 0.793 & 0.781 \\
\hline Vit. $B_{1}$ & 0.774 & 0.788 & 0.795 & 0.803 & 0.790 \\
\hline Vit. C & 0.787 & 0.799 & 0.805 & 0.814 & 0.801 \\
\hline Vit. E & 0.792 & 0.804 & 0.810 & 0.817 & 0.805 \\
\hline Vit. $B_{1}+$ Vit. C+Vit. E & 0.798 & 0.813 & 0.817 & 0.824 & 0.813 \\
\hline Yeast & 0.780 & 0.795 & 0.799 & 0.809 & 0.796 \\
\hline Mean (A) & 0.783 & 0.796 & 0.801 & 0.810 & 0.797 \\
\hline L.S.D. at $5 \%$ & \multicolumn{2}{|c|}{ A : 0.004} & B : 0.004 & \multicolumn{2}{|r|}{$\mathrm{AB}: 0.008$} \\
\hline \multicolumn{6}{|c|}{$3^{\text {rd }}$ Cut } \\
\hline Control & 0.758 & 0.771 & 0.780 & 0.787 & 0.775 \\
\hline Vit. $B_{1}$ & 0.768 & 0.780 & 0.785 & 0.796 & 0.783 \\
\hline Vit. C & 0.780 & 0.791 & 0.796 & 0.806 & 0.793 \\
\hline Vit. E & 0.786 & 0.797 & 0.803 & 0.815 & 0.800 \\
\hline Vit. $B_{1}+$ Vit. C+Vit. E & 0.793 & 0.804 & 0.813 & 0.818 & 0.808 \\
\hline Yeast & 0.774 & 0.785 & 0.791 & 0.801 & 0.788 \\
\hline Mean (A) & 0.777 & 0.788 & 0.795 & 0.804 & 0.791 \\
\hline L.S.D. at $5 \%$ & \multicolumn{2}{|c|}{ A : 0.005} & B : 0.003 & \multicolumn{2}{|r|}{$\mathrm{AB}: 0.006$} \\
\hline
\end{tabular}


M.A.H. Abdou et al.

Table 11. Effect of plant densities and some vitamins (vit. $B_{1}$, vit. $C$ and vit. $E$ ) and yeast treatments on carotenoids of sweet basil (Ocimum basilicum, L.) plants during the first season.

\begin{tabular}{|c|c|c|c|c|c|}
\hline \multicolumn{6}{|c|}{$1^{\text {st }}$ Cut } \\
\hline \multirow{2}{*}{ Treatments (B) } & \multicolumn{5}{|c|}{ Plant densities $/ 14.5 \mathrm{~m}^{2}$ (A) } \\
\hline & 145 & 105 & 80 & 65 & Mean (B) \\
\hline Control & 0.761 & 0.772 & 0.790 & 0.787 & 0.778 \\
\hline Vit. $B_{1}$ & 0.772 & 0.781 & 0.788 & 0.796 & 0.784 \\
\hline Vit. C & 0.782 & 0.793 & 0.802 & 0.810 & 0.797 \\
\hline Vit. E & 0.787 & 0.798 & 0.796 & 0.817 & 0.800 \\
\hline Vit. $B_{1}+$ Vit. C +Vit. E & 0.792 & 0.804 & 0.812 & 0.824 & 0.808 \\
\hline Yeast & 0.778 & 0.787 & 0.793 & 0.803 & 0.790 \\
\hline Mean (A) & 0.779 & 0.789 & 0.798 & 0.805 & 0.793 \\
\hline L.S.D. at $5 \%$ & \multicolumn{2}{|c|}{ A : 0.003} & B : 0.004 & \multicolumn{2}{|r|}{$\mathrm{AB}: 0.008$} \\
\hline \multicolumn{6}{|c|}{$2^{\text {nd }}$ Cut } \\
\hline Control & 0.777 & 0.783 & 0.792 & 0.800 & 0.788 \\
\hline Vit. $B_{1}$ & 0.782 & 0.795 & 0.803 & 0.810 & 0.798 \\
\hline Vit. C & 0.794 & 0.807 & 0.812 & 0.820 & 0.808 \\
\hline Vit. E & 0.799 & 0.811 & 0.819 & 0.823 & 0.813 \\
\hline Vit. $B_{1}+$ Vit. C+Vit. E & 0.805 & 0.820 & 0.824 & 0.830 & 0.820 \\
\hline Yeast & 0.787 & 0.802 & 0.807 & 0.815 & 0.803 \\
\hline Mean (A) & 0.791 & 0.803 & 0.810 & 0.816 & 0.805 \\
\hline L.S.D. at $5 \%$ & \multicolumn{2}{|c|}{ A : 0.004} & B : 0.005 & \multicolumn{2}{|r|}{$\mathrm{AB}: 0.010$} \\
\hline \multicolumn{6}{|c|}{$3^{\text {rd }}$ Cut } \\
\hline Control & 0.767 & 0.779 & 0.788 & 0.795 & 0.782 \\
\hline Vit. $B_{1}$ & 0.778 & 0.787 & 0.793 & 0.803 & 0.790 \\
\hline Vit. C & 0.787 & 0.799 & 0.803 & 0.813 & 0.801 \\
\hline Vit. E & 0.794 & 0.804 & 0.810 & 0.820 & 0.807 \\
\hline Vit. $B_{1}+$ Vit. C+Vit. E & 0.801 & 0.812 & 0.820 & 0.825 & 0.815 \\
\hline Yeast & 0.782 & 0.793 & 0.799 & 0.808 & 0.796 \\
\hline Mean (A) & 0.785 & 0.796 & 0.802 & 0.811 & 0.799 \\
\hline L.S.D. at $5 \%$ & \multicolumn{2}{|c|}{ A : 0.005} & B : 0.004 & \multicolumn{2}{|r|}{$\mathrm{AB}: 0.008$} \\
\hline
\end{tabular}


Table 12. Effect of plant densities and some vitamins (vit. $B_{1}$, vit. $C$ and vit. $E$ ) and yeast treatments on carotenoids of sweet basil (Ocimum basilicum, L.) plants during the second season.

\begin{tabular}{|c|c|c|c|c|c|}
\hline \multicolumn{6}{|c|}{$1^{\text {st }}$ Cut } \\
\hline \multirow{2}{*}{ Treatments (B) } & \multicolumn{5}{|c|}{ Plant densities $/ 14.5 \mathrm{~m}^{2}$ (A) } \\
\hline & 145 & 105 & 80 & 65 & Mean (B) \\
\hline Control & 0.784 & 0.795 & 0.814 & 0.811 & 0.801 \\
\hline Vit. $B_{1}$ & 0.795 & 0.804 & 0.812 & 0.820 & 0.808 \\
\hline Vit. C & 0.805 & 0.817 & 0.826 & 0.834 & 0.821 \\
\hline Vit. E & 0.811 & 0.822 & 0.820 & 0.842 & 0.824 \\
\hline Vit. $B_{1}+$ Vit. $C+$ Vit. E & 0.816 & 0.828 & 0.836 & 0.849 & 0.832 \\
\hline Yeast & 0.801 & 0.811 & 0.817 & 0.827 & 0.814 \\
\hline Mean (A) & 0.802 & 0.813 & 0.822 & 0.829 & 0.817 \\
\hline L.S.D. at $5 \%$ & \multicolumn{2}{|c|}{ A : 0.004} & B : 0.005 & \multicolumn{2}{|r|}{$\mathrm{AB}: 0.010$} \\
\hline \multicolumn{6}{|c|}{$2^{\text {nd }}$ Cut } \\
\hline Control & 0.800 & 0.806 & 0.816 & 0.824 & 0.812 \\
\hline Vit. $B_{1}$ & 0.805 & 0.819 & 0.827 & 0.834 & 0.822 \\
\hline Vit. C & 0.818 & 0.831 & 0.836 & 0.845 & 0.832 \\
\hline Vit. E & 0.823 & 0.835 & 0.844 & 0.848 & 0.837 \\
\hline Vit. $B_{1}+$ Vit. C+Vit. E & 0.829 & 0.845 & 0.849 & 0.855 & 0.845 \\
\hline Yeast & 0.811 & 0.826 & 0.831 & 0.839 & 0.827 \\
\hline Mean (A) & 0.815 & 0.827 & 0.834 & 0.840 & 0.829 \\
\hline L.S.D. at $5 \%$ & \multicolumn{2}{|c|}{ A : 0.005} & B : 0.005 & \multicolumn{2}{|r|}{$\mathrm{AB}: 0.010$} \\
\hline \multicolumn{6}{|c|}{$3^{\text {rd }}$ Cut } \\
\hline Control & 0.790 & 0.802 & 0.812 & 0.819 & 0.805 \\
\hline Vit. $B_{1}$ & 0.801 & 0.811 & 0.817 & 0.827 & 0.814 \\
\hline Vit. C & 0.811 & 0.823 & 0.827 & 0.837 & 0.825 \\
\hline Vit. E & 0.818 & 0.828 & 0.834 & 0.845 & 0.831 \\
\hline Vit. $B_{1}+$ Vit. C+Vit. E & 0.825 & 0.836 & 0.845 & 0.850 & 0.839 \\
\hline Yeast & 0.805 & 0.817 & 0.823 & 0.832 & 0.820 \\
\hline Mean (A) & 0.809 & 0.820 & 0.826 & 0.835 & 0.823 \\
\hline L.S.D. at $5 \%$ & \multicolumn{2}{|c|}{ A : 0.005} & B : 0.006 & \multicolumn{2}{|r|}{$\mathrm{AB}: 0.012$} \\
\hline
\end{tabular}


significantly increased the leaves contents of chlorophyll a, b and carotenoids during the three cuts in both seasons as compared with control treatments. Among these five treatments, the treatment of vit. $\mathrm{B}_{1}+$ vit. $\mathrm{C}+$ vit. $\mathrm{E}$ resulted the highest contents of chlorophyll a, b and carotenoids. The positive effect of vitamins on contents of the pigments of sweet basil was found by Abd El-Salam (2014).

The interaction between plant densities, some vitamins and active yeast treatments was significant for chlorophyll a, b and carotenoids during the three cuts in both seasons. The highest contents of chlorophyll a and carotenoids were obtained with lower density ( 65 plants $/ 14.5 \mathrm{~m}^{2}$ ) plus vit. $\mathrm{B}_{1}+$ vit. $\mathrm{C}+$ vit. $\mathrm{E}$ or vit. $\mathrm{E}$ during the three cuts in both seasons or plant density 80 plants/14.5 $\mathrm{m}^{2}$ in combination with vit. $\mathrm{B}_{1}+$ vit. $\mathrm{C}+$ vit. $\mathrm{E}$ during the second and third cuts in both seasons as clearly shown in Tables $(7,8,11$ and 12). The highest contents of chlorophyll $\mathrm{b}$, during the three cuts in both seasons, were obtained with 65 and 80 plants $/ 14.5 \mathrm{~m}^{2}$ in combination with vit. $\mathrm{B}_{1}+$ vit. $\mathrm{C}+$ vit. $\mathrm{E}$ or 65 plants $/ 14.5 \mathrm{~m}^{2}$ with vit. $\mathrm{E}$ as clearly shown in Tables (9 and 10).

\section{2- N, P and $K \%$ :}

Data presented in Tables (13, 14 and 15) cleared that sweet basil planted at 65 plants $/ 14.5 \mathrm{~m}^{2}$ recorded significantly highest percentages of nitrogen, phosphorus and potassium in the dry herb in both seasons comparing with other densities treatments. Similar results were obtained by El-Shaer (1986) on fennel and Badran et al. (2003) on anise plants who found that the elements \% (NPK) in the dry herb was slightly increased by decreasing plant density.

Data in Tables (13, 14 and 15) mentioned that all used five treatments (vit. $\mathrm{B}_{1}$, vit. $\mathrm{C}$, vit. $\mathrm{E}$, vit. $\mathrm{B}_{1}+$ vit. $\mathrm{C}+$ vit. $\mathrm{E}$ and active yeast) significantly increased $\mathrm{N}$. $\mathrm{P}$ and $\mathrm{K} \%$ in both seasons comparing with control.
The treatment of active yeast significantly increased the percentages of $\mathrm{N}$, $\mathrm{P}$ and $\mathrm{K}$ comparing with other treatments.

Active yeast had positive effect on percentages of $\mathrm{N}, \mathrm{P}$ and $\mathrm{K}$ as mentioned by Abd-El-Salam (2014) on basil plants, ElNady (2015) on lemongrass and Khaled et al. (2014) on marjoram plants.

The interaction between main and sub plots $(\mathrm{A} \times \mathrm{B})$ treatments was significant for $\mathrm{N}$, $\mathrm{P}$ and $\mathrm{K} \%$ in both seasons (Tables, 13, 14 and 15). The best interaction treatments for $\mathrm{N}, \mathrm{P}$ and $\mathrm{K} \%$ were obtained with 65 plants $/ 14.5 \mathrm{~m}^{2}$ in combination with active yeast or vit. $B_{1}+$ vit. $C+$ vit. $E$.

\section{REFERENCES}

Abd El-Salam, Nora M.K. (2014). Response of Sweet Basil Plants to Some Agricultural Treatments. Ph.D. Thesis, Fac. Agric., Minia Univ.

Abdou, M.A.H.; Badran, F.S.; El-Sayed, A.A.; Taha, R.A. and Abd-El-Salam, N.M.K. (2014). Response of sweet basil plants to some agricultural treatments. Minia J. of Agric. Res. \& Develop., 34(1):21-31.

Arabasi, O. and Bayran, E. (2004). The effect of nitrogen fertilization and different plant densities on some agronomic and technologic parameters of basil. J. of Agronomy, 3(4):255-262.

Atghaei, M.S.; Rajabi, A. and Hemayaty, S.S. (2015). Investigating the effect of bush density on the yield and leaf essence of green basil (Ocimum basilicum). Inter. J. Res. Studies in Agricultural Sciences, 1(1):11-17.

Badran, F.S.; Abdalla, N.M.; Aly, M.K. and Ibrahim, S.M. (2007). Response of fennel plants to seedling rate and partial replacement of mineral NPK by biofertilization treatments, Pro. of the $8^{\text {th }}$ African Crop. Sci. Conf., El-Minia, Egypt, 27-31, Oct., 2007, 8(1):417-422. 
Table 13. Effect of plant densities and some vitamins (vit. $B_{1}$, vit. $C$ and vit. $E$ ) and yeast treatments on nitrogen percentage of sweet basil (Ocimum basilicum, L.) plants during two seasons.

\begin{tabular}{|c|c|c|c|c|c|}
\hline \multicolumn{6}{|c|}{ First season } \\
\hline \multirow{2}{*}{ Treatments (B) } & \multicolumn{5}{|c|}{ Plant densities /14.5 $\mathrm{m}^{2}$ (A) } \\
\hline & 145 & 105 & 80 & 65 & Mean (B) \\
\hline Control & 1.830 & 1.854 & 1.879 & 1.893 & 1.864 \\
\hline Vit. $B_{1}$ & 1.850 & 1.876 & 1.899 & 1.914 & 1.885 \\
\hline Vit. C & 1.880 & 1.916 & 1.929 & 1.944 & 1.917 \\
\hline Vit. E & 1.865 & 1.891 & 1.914 & 1.929 & 1.900 \\
\hline Vit. $B_{1}+$ Vit. C+Vit. E & 1.876 & 1.902 & 1.925 & 1.939 & 1.911 \\
\hline Yeast & 1.887 & 1.912 & 1.936 & 1.949 & 1.921 \\
\hline Mean (A) & 1.865 & 1.892 & 1.914 & 1.928 & \\
\hline L.S.D. at $5 \%$ & \multicolumn{2}{|c|}{$A: 0.005$} & B : 0.006 & \multicolumn{2}{|r|}{$\mathrm{AB}: 0.012$} \\
\hline \multicolumn{6}{|c|}{ Second season } \\
\hline Control & 1.834 & 1.866 & 1.890 & 1.903 & 1.873 \\
\hline Vit. $B_{1}$ & 1.856 & 1.889 & 1.914 & 1.926 & 1.896 \\
\hline Vit. C & 1.890 & 1.924 & 1.947 & 1.958 & 1.930 \\
\hline Vit. E & 1.875 & 1.908 & 1.932 & 1.943 & 1.915 \\
\hline Vit. $B_{1}+$ Vit. C+Vit. E & 1.891 & 1.936 & 1.956 & 1.968 & 1.938 \\
\hline Yeast & 1.899 & 1.939 & 1.958 & 1.986 & 1.946 \\
\hline Mean (A) & 1.874 & 1.910 & 1.933 & 1.986 & \\
\hline L.S.D. at $5 \%$ & \multicolumn{2}{|c|}{ A : 0.004} & B : $0.00 \subseteq$ & \multicolumn{2}{|r|}{$\mathrm{AB}: 0.018$} \\
\hline
\end{tabular}

Table 14. Effect of plant densities and some vitamins (vit. $B_{1}$, vit. $C$ and vit. $E$ ) and yeast treatments on phosphorus percentage of sweet basil (Ocimum basilicum, L.) plants during two seasons.

\begin{tabular}{|c|c|c|c|c|c|}
\hline \multicolumn{6}{|c|}{ First season } \\
\hline \multirow{2}{*}{ Treatments (B) } & \multicolumn{5}{|c|}{ Plant densities $/ 14.5 \mathrm{~m}^{2}$ (A) } \\
\hline & 145 & 105 & 80 & 65 & Mean (B) \\
\hline Control & 0.116 & 0.131 & 0.146 & 0.158 & 0.138 \\
\hline Vit. $\mathbf{B}_{1}$ & 0.140 & 0.162 & 0.173 & 0.182 & 0.164 \\
\hline Vit. C & 0.152 & 0.175 & 0.185 & 0.198 & 0.178 \\
\hline Vit. E & 0.133 & 0.151 & 0.163 & 0.169 & 0.154 \\
\hline Vit. $B_{1}+$ Vit. C+Vit. E & 0.161 & 0.186 & 0.198 & 0.218 & 0.191 \\
\hline Yeast & 0.171 & 0.201 & 0.210 & 0.218 & 0.200 \\
\hline Mean (A) & 0.146 & 0.168 & 0.179 & 0.191 & \\
\hline L.S.D. at $5 \%$ & \multicolumn{2}{|c|}{ A : 0.002} & $\mathrm{~B}: 0.003$ & \multicolumn{2}{|r|}{$\mathrm{AB}: 0.006$} \\
\hline \multicolumn{6}{|c|}{ Second season } \\
\hline Control & 0.125 & 0.140 & 0.151 & 0.162 & 0.145 \\
\hline Vit. $B_{1}$ & 0.141 & 0.165 & 0.177 & 0.185 & 0.167 \\
\hline Vit. C & 0.155 & 0.180 & 0.186 & 0.201 & 0.181 \\
\hline Vit. E & 0.135 & 0.155 & 0.166 & 0.171 & 0.157 \\
\hline Vit. $B_{1}+$ Vit. C+Vit. E & 0.168 & 0.189 & 0.211 & 0.225 & 0.198 \\
\hline Yeast & 0.179 & 0.215 & 0.218 & 0.231 & 0.211 \\
\hline Mean (A) & 0.151 & 0.174 & 0.185 & 0.196 & \\
\hline L.S.D. at $5 \%$ & \multicolumn{2}{|c|}{$A: 0.001$} & B : 0.004 & & $\mathrm{AB}: 0.008$ \\
\hline
\end{tabular}


Table 15. Effect of plant densities and some vitamins (vit. $B_{1}$, vit. $C$ and vit. $E$ ) and yeast treatments on potassium percentage of sweet basil (Ocimum basilicum, L.) plants during two seasons.

\begin{tabular}{|c|c|c|c|c|c|}
\hline \multicolumn{6}{|c|}{ First season } \\
\hline \multirow{2}{*}{ Treatments (B) } & \multicolumn{5}{|c|}{ Plant densities $/ 14.5 \mathrm{~m}^{2}$ (A) } \\
\hline & 145 & 105 & 80 & 65 & Mean (B) \\
\hline Control & 1.319 & 1.349 & 1.375 & 1.391 & 1.359 \\
\hline Vit. $B_{1}$ & 1.347 & 1.370 & 1.395 & 1.415 & 1.382 \\
\hline Vit. C & 1.382 & 1.435 & 1.448 & 1.456 & 1.430 \\
\hline Vit. E & 1.371 & 1.421 & 1.435 & 1.441 & 1.417 \\
\hline Vit. $B_{1}+$ Vit. C+Vit. E & 1.399 & 1.449 & 1.459 & 1.478 & 1.446 \\
\hline Yeast & 1.419 & 1.469 & 1.472 & 1.487 & 1.462 \\
\hline Mean (A) & 1.373 & 1.416 & 1.431 & 1.445 & \\
\hline L.S.D. at $5 \%$ & \multicolumn{2}{|c|}{ A : 0.002} & B : 0.006 & \multicolumn{2}{|r|}{ AB : 0.012} \\
\hline \multicolumn{6}{|c|}{ Second season } \\
\hline Control & 1.323 & 1.354 & 1.381 & 1.398 & 1.364 \\
\hline Vit. $B_{1}$ & 1.350 & 1.374 & 1.400 & 1.421 & 1.386 \\
\hline Vit. C & 1.385 & 1.439 & 1.453 & 1.462 & 1.435 \\
\hline Vit. E & 1.374 & 1.425 & 1.441 & 1.448 & 1.422 \\
\hline Vit. $B_{1}+$ Vit. C+Vit. E & 1.403 & 1.454 & 1.465 & 1.484 & 1.452 \\
\hline Yeast & 1.423 & 1.474 & 1.478 & 1.494 & 1.467 \\
\hline Mean (A) & 1.376 & 1.420 & 1.436 & 1.451 & \\
\hline L.S.D. at $5 \%$ & \multicolumn{2}{|c|}{ A : 0.001} & B : 0.005 & & $\mathrm{AB}: 0.010$ \\
\hline
\end{tabular}

Badran, F.S.; Attia, F.A.; Ahmed, E.T. and Soliman, H.A. (2003). Effect of chemical and biological fertilization on growth, yield and oil production of anise (Pimpinella anisum, L.) plants. II-Effect of NP mineral/biofertilization and micronutrient treatments. Proc. EgyptianSyrian $1^{\text {st }}$ Conf., Minia Univ.

Badran, F.S. and Hafez, M.H. (2002). Effect of plant density, planting date, nitrogen fertilization sources and some nutrients on growth, flowering, seed yield and soil of Nigella sativa, L. plants.

Bowes, K.M. and Zheljazkov, V.D. (2004). Factors affecting yields and essential oil quality of Ocimum sanctum, L. and Ocimum basilicum, Cultivars. J. A.M. Soc. Hort. Sci., 129:789-794.

British Pharmacopoeia (1963). Determination of Volatile Oil Drugs. The Pharmaceutical Press, London.

Chapman, H.D. and Pratt, P.F. (1975). Methods of Analysis for Soil, Plant and
Water. Calif. Univ. Division of Agric. Sci., 172-174.

Cottenie, A.; Verloo, M.; Velghe, M. and Camerlynck, R. (1982). Chemical Analysis of Plant and Soil. Laboratory of Analytical and Agro Chemistry. State Univ., Ghent, Belgium.

Dadvand, M.R.; Naghdibadi, H. and Nasri, M. (2009). Variation of essential oil and yield in Ocimum affected by density and nitrogen fertilizers. Iranian research Journal of Medicinal Plant. 27:70-80.

El-Gendy, S. A.; Hosni, A. M.; Ahmed, S. S. and Saber, R. M. (2001). Sweet basil (Ocimum basilicum L.) productivity under different organic fertilization and inter-plant spacing levels in a newly reclaimed land in Egypt. Ann. Agric. Sci. Ain Shams Univ., 46(1):319-338.

El-Nady, M.K. (2015). Physiological Studies on Lemongrass Plants. M.Sc. Thesis, Fac. Agric. Minia Univ. 
El-Shaer, S.T. (1986). Effect of Plant Spacing and Growth Regulators on Growth, Seed Yield and Volatile Oil of Fennel Plant. M.Sc. Thesis, Fac. Agric., Zagazig Univ.

Helmy, T.A. (2016). Influence of Agricultural Treatments on Cumin Plant. Ph.D. Thesis, Fac. Agric., Minia Univ. Egypt.

Jackson, M.L. (1973). Soil Chemical Analysis Englewood Cliffs., New Prentice-Hall INC., New York.

Katar, D. and Gurbuz, B. (2008). The effect of different plant densities and nitrogen doses on drug leaf yield and some features of lemon balm (Melissa officinalis, L.). Tarim Bilimleri Dergisi, 14(1):78-81.

Khafi, M. (2003). Basil-Production and Processing. Ferdowsi Univ. Mashhad Publication. Mashhad, Iran 195 pp.

Khaled, S.A.; Abdella, E.M. and Mohamed, G.F. (2014). Response of growth, chemical composition, anatomical structure, antioxidant and antimicrobial activity of marjoram to yeast and methionine. International Journal of Academic Research, 6(1):18-30.

Moran, R. (1982). Formula determination of chlorophylls pigments extracted with $\mathrm{N}$ $\mathrm{N}$ dimethyl-formamide. Plant Physiological., 69:1376-1381.
MSTAT-C (1986). A Microcomputer Program for the Design, Management and Analysis of Agronomic Research Experiments (Version 4.0), Michigan State Univ., U.S.

Nassar, M.A.; Mohamed, U.E. and Azoz, S.N. (2015). Influence of foliar spray with yeast extract on vegetative growth, yield of fresh herb, anatomical structure, composition of volatile oil and seed yield components of basil plant (Ocimum basilicum, L.). Int. J. of Advanced Research, 3(10):978-993.

Omar, E.A.; Hussein, M.S.; Osman, A.R.; Sewedan, E.; Elgohary, A. and Salman, A.M. (2016). Response of basil essential oil to cultivation date and organic fertilization. Int. J. of Pharm. Tech. Research, 9(5):86-98.

Ram, M.; Ram, D.; Nagvi, A.A. and Kumar, S. (2002). Effect of plant density and harvesting time on the yield and the quality of essential oil on Ocimum spp. J. of Medicinal and Aromatic Plant Sci., 24(2):393-396.

Salman, A.S. (2006). Effect of biofertilization on Ocimum basilicum, L. plant. Egypt J. Agric. Res., 79(2):587606.

Wilde, S.A.; Covey, R.P.; Lyer, J.C. and Voigt, G.K. (1985). Soil and Plant Analysis for Tree Culture. Oxford, IBH. Publishing Co., New Delhi, India.

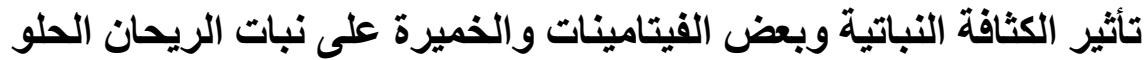

$$
\begin{aligned}
& \text { ب - إنتاجية الزيت العطري والمكونات الكيماوية والفية }
\end{aligned}
$$

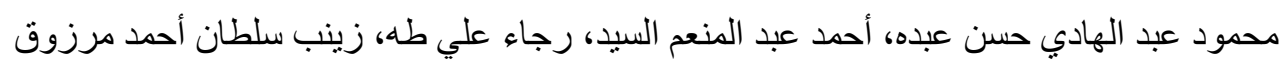

$$
\begin{aligned}
& \text { قسم البساتين، كلية الزراعة، جامعة المنيا، مصر. }
\end{aligned}
$$

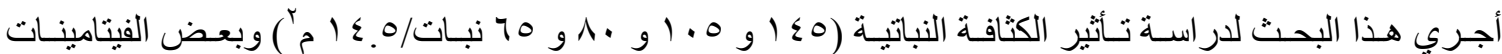

$$
\begin{aligned}
& \text { (فيتامين ب, ، فيتامين ج ، فيتامين هـ الخميرة النشطة والكنترول) على إنتاجية الزيت الطيار و التحليل الكيمـاوي لنبات النيات } \\
& \text { الريحان. }
\end{aligned}
$$

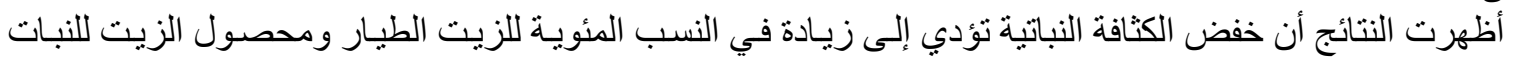

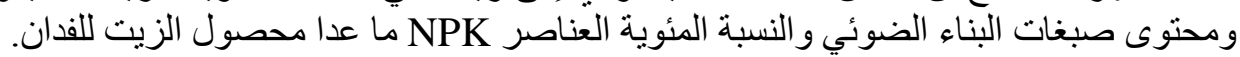




\section{M.A.H. Abdou et al.}

كل المعاملات المستخدمة من الفيتامينات و الخميرة النشطة تؤدي إلى زيادة معنوية في صفات إنتاجية الزيت الطيار و التحليل الكيماوي.

معاملة الخميرة يليها معاملة الفيتامينات معا (فيتامين ب, + فيتامين ج + فيتامين هـ) سجلت أعلى القيم في هذا الثأن.

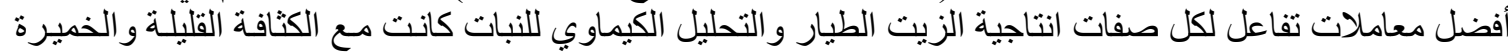

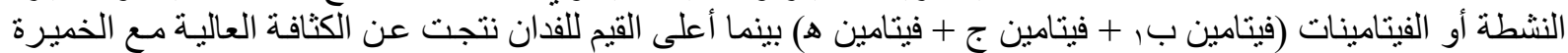
النشطة أو فيتامين ب, + فيتامين ج + فيتامين هـ 